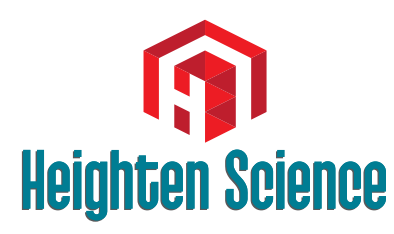

P U B L I C I T I O N S Corporation

*Address for Correspondence: Awad Magbri, MD, FACP, Toledo Vascular Access Center Toledo, OH, USA, Tel: 815-520-8211; Email: elmagbri@hotmail.com

Submitted: 13 December 2018

Approved: 27 December 2018

Published: 28 December 2018

Copyright: @ 2018 Magbri A, et al. This is an open access article distributed under the Creative Commons Attribution License, which permits unrestricted use, distribution, and reproduction in any medium, provided the original work is properly cited

Check for updates
Opinion

\section{Navigation in the land of Scarcity}

\author{
Awad Magbri* and Mariam El-Magbri \\ Toledo Vascular Access Center, Toledo, $\mathrm{OH}, \mathrm{USA}$
}

Delivering health care is a complex task that marginalized a portion of the population intentionally or unintentionally. Discrepancy in health care providing to the intended patients is sometimes accompanied by unintended collateral damage to the bystanders who desperately needing our help and assistance.

I was called to help in the management of a 75-year old Caucasian male with history of ischemic heart disease requiring percutaneous coronary intervention (PCI) for 2-vessel disease treated with stent placement, hyperlipidemia, chronic obstructive pulmonary disease, peripheral vascular disease, and diastolic heart failure with preserved ejection fraction of $50-55 \%$. The patient was admitted this time with community acquired pneumonia resulting in hypoxemic respiratory failure demanding initiation of mechanical ventilation and tracheostomy placement.

The hospital course is complicated by sepsis with acute kidney injury (AKI) and nephrotic range proteinuria, the cause of which was not clearly evident. The patient required 3-sessions of hemodialysis for fluid overload and deteriorating kidney function before he was taken off hemodialysis. Most of his illnesses can be explained on the basis of history, examination, and laboratory investigation. However, the nephrotic range proteinuria cannot be explained by the above measures. At the same time kidney biopsy may not be possible during the acute phase to unravel the underlying pathology. This will create a significant management challenge for the caring physician. Had kidney biopsy been done in this patient with all the attended complications in mind, a diagnosis of membranous nephropathy or some other primary kidney disease could have been confirmed. If for the sake of discussion, a primary membranous nephropathy was diagnosed, then we would be obliged to provide specific treatment in the form of steroid plus cyclophosphamide or rituximab.

One would be struggling with justification of potentially toxic treatment for a disease that might not be relevant to the overall prognosis of this patient. On the other hand, you could be persuaded that if you provide treatment for the membranous nephropathy in this patient, you could change his recovery course and prognosis. The question of renal biopsy and treatment of underlying disease in a patient with multitude of comorbid conditions cannot be settled effortlessly. We should all tussle with the notion that treating patients and not disease processes are the path of action that we should take to influence the overall prognosis of patients under our care.

This scenario looks familiar to most of us. Our patients today, have compounding comorbid conditions, with sometimes unsalvageable underlying disease. Because of the gap in our understanding of the pathophysiology of most disease processes that affect the unfortunate patients, compounded with the lack of diligent and honest conversation with the family, all of which, would force us sometimes to take the easy way out and resist the temptations of taking drastic measures for diagnosis and specific treatment of the underlying diseases. Without knowing the limitations of today's medicine we can pander in futile and drastic measures, the consequences of which, may be more harmful to the patients under our care. 
We, as professionals, can cure only tiny fraction of maladies, but healing the underlying disease should be a major part of our assignment that we aspire to achieve. Our ability to predict what would happen next succumbed to the acknowledgment of uncertainty. Rationing the health care service to benefit a major part of patient's population are steps sometimes need to be taken [1-3]. Defensive medicine is bad for patients, doctors, and the health system alike. The United States does have a comparatively high level of imaging units and patient surgeries compared with other industrialized nations $[4,5]$. Effective Government's regulations of health system to control care spending and curtail useless unsubstantiated costs are urgently needed [6-8].

On the other hand, rationing health care for many lifestyle choices such as smoking, drinking, unhealthy eating habits, overuse and abuse of drugs, unmasked patient safety concerns such as domestic and community violence, reckless driving, etc., needed to be seriously addressed [8].

As active participant in the scientific community, we are required to articulate the best-practice guidelines based on scientific evidence that everybody should follow to cut unjustifiable costs and at the same time avoiding spending not based on demonstrable, value-added, cost-effective principles [8]. We need to challenge the traditional art of medicine that is based on endless increase in health care spending. Throwing money on the problem is not going to solve it. Smart medicine will stimulate our solicitous broad-based decisions and rationalize the limited resources that we desperately needed to make a change.

\section{References}

1. Aaron HJ, Schwartz WB. The painful prescription: rationing hospital. 1984; Ref.: https://goo.gl/B2mHJT

2. Somers A. The rationalization of health services: a universal priority. Inquiry. 1971; 8: 48-60. Ref.: https://goo.gl/fhybXW

3. Mushkin SJ. Health as an investment. J Polit Econ 1962; 70: 129-157. Ref.: https://goo.gl/2hnurh

4. Vladeck BC. Everything new is old again. Health Aff (Millwood). 2004; 23: VAR108-111. Ref.: https://goo.gl/Rp2h7R

5. Ref.: https://goo.gl/zHEssK

6. Ref.: https://goo.gl/c7PgMt

7. Ref.: https://goo.gl/A2r285

8. Morone JA. The bias of American Politics: rationing health care in a weak state. 1992. U Pa L Rev 150: 1923-1938. Ref.: https://goo.gl/DGssQJ 